\title{
The effect of eight weeks of interval training and quercetin nanoliposome supplementation on NF- $\kappa B$ and FGF-2 gene expression in the heart tissue of myocardial infarction rats
}

\author{
Samira Zaheri Abdehvand ${ }^{1}$, Khosro Jalali Dehkordi ${ }^{* *}{ }^{(\mathbb{D}}$, Farzaneh Taghian ${ }^{2}$ \\ ${ }^{1}$ PhD Student, Department of Physical Education and Sport Sciences, Isfahan (Khorasgan) Branch, Islamic Azad University, \\ Isfahan, Iran \\ ${ }^{2}$ Assistant Professor, Department of Physical Education and Sport Sciences, Isfahan (Khorasgan) Branch, Islamic Azad University, \\ Isfahan, Iran
}

*Corresponding Author: Khosro Jalali Dehkordi, Department of Physical Education and Sport Sciences, Isfahan (Khorasgan) Branch, Islamic Azad University, Isfahan, Iran, Postcode: 81551-39998, Tele: +989131854997, Fax: +98031-353-54135, Email: khosrojalali@gmail.com

\begin{abstract}
Background and aims: The activation of inflammatory reactions is essential immediately after the onset of myocardial infarction (MI). On the other hand, the path of inflammatory activators should be controlled to prevent the recurrence of $\mathrm{MI}$. In this vein, the aim of this study was to examine the effect of eight weeks of interval training and quercetin nanoliposome consumption on nuclear factor kappa B (NF-kB) and fibroblast growth factor 2 (FGF-2) gene expression in the heart tissue of MI rats.

Methods: In this experimental study, 30 male rats, aged approximately 6-8 weeks, were randomly divided into five groups including MI + training, $\mathrm{Ml}+$ supplement, $\mathrm{MI}+$ training + supplement, healthy control, and $\mathrm{MI}$. The $\mathrm{MI}$ was induced by the subcutaneous injection of isoprenaline hydrochloride at a dose of $80 \mathrm{mg} / \mathrm{kg}$. Quercetin was daily administered at a dose of $0.25 \mathrm{mg}$ for 8 weeks, and interval training was performed 5 days a week. NF-KB and FGF-2 gene expressions were measured by the real-time polymerase chain reaction. Finally, data were analyzed using the one-way analysis of variance (ANOVA) and Tukey's post hoc tests $(P<0.05)$.

Results: Training significantly increased NF- $\mathrm{kB}$ gene expression in the Ml+ training, $\mathrm{MI}+$ supplement, and $\mathrm{MI}+$ training + supplement groups $(P=0.001)$ while significantly decreasing FGF-2 gene expression in the MI group $(P=0.04)$.

Conclusion: Interval training may prevent the negative effects of $\mathrm{MI}$ by reducing pro-inflammatory gene expression in the heart tissue, leading to improved cardiovascular function and the prevention of this disease.

Keywords: High-intensity interval training, Nuclear factor kappa B, Fibroblast growth factor-2, Quercetin supplementation, Myocardial infarction
\end{abstract}

Received: 13 September 2021, Accepted: 29 November 2021, ePublished: 26 January 2022

\section{Introduction}

Myocardial infarction (MI) is the damage or loss of an area of the myocardium that results from the blockage of blood supply to that area, which eventually leads to the death of the heart tissue (1). Following the death of myocytes and the loss of capillaries in and around the area affected by the infarction, active myocardial remodeling continues, increasing the signals that induce the vascular growth and remodeling of the left ventricle and the fibrosis tissue and resulting in the left ventricle dilation and thus heart failure. The growth of new blood vessels and capillaries after the onset of infarction seems to be a necessary process to be able to maintain left ventricular hypertrophy in the living part (2). Increasing the density of skeletal and cardiac muscle capillaries is called the process of angiogenesis, which requires the proliferation and migration of capillary endothelial cells (3).

The most important factor in the process of angiogenesis is the fibroblast growth factor 2 (FGF-2), which is involved in angiogenesis, wound healing, and embryonic development. FGFs play an important role in the proliferation and differentiation processes of body cells and tissues. Decreased FGF gene expression leads to decreased muscle mass, followed by decreased muscle strength, and ultimately, decreased physical function (4). In general, 20 types of FGF have so far been identified, of which FGF-1 and FGF-2 are the primary growth factors that have been studied for stimulating vascularization. These two agents have chemotoxic and mitogenic effects on endothelial cells, fibroblasts, and many other cells. Different factors exist for stimulating vascularization acting at different stages. It has been reported that FGF2 and vascular endothelial growth factor (VEGF) play an important role in the early stages of vascularization by binding to their specific receptors (5). Given that MI is caused by the impaired blood supply and oxygen supply

(C) 2022 The Author(s); Published by Shahrekord University of Medical Sciences. This is an open-access article distributed under the terms of the Creative Commons Attribution License (http://creativecommons.org/licenses/by/4.0), which permits unrestricted use, distribution, and reproduction in any medium, provided the original work is properly cited. 
to the heart muscle cells, it is important for evaluating the hypoxia condition. A dramatic increase in hypoxiainduced protein and mRNA (nuclear factor kappa B, NF$\kappa \mathrm{B})$ occurs under these conditions.

$\mathrm{NF}-\kappa \mathrm{B}$ is induced by many factors including inflammatory cytokines (interleukin-1 NTFa), and oxidative stress $\left(\mathrm{H}_{2} \mathrm{O}_{2}\right.$ mitogen-activated compounds). Under cellular resting conditions, NF- $\kappa \mathrm{B}$ lies within the cytoplasm as a complex attached to the $\kappa \mathrm{B}$ inhibitor (I $\kappa \mathrm{B}$ ) that activates various stimuli in the intracellular signal pathways, thereby activating I $\mathrm{B}$ kinase (IKK). Activated IKK by the phosphorylation and ubiquitination of the $\kappa B$ inhibitor destroys the inhibitor. Then, NF- $\kappa \mathrm{B}$ is released for transmission into the nucleus and regulates the expression of NF- $\kappa \mathrm{B}$-dependent genes (6).

Exercise as a non-pharmacological intervention plays an essential role in preventing and improving the treatment of cardiovascular patients. Among the types of exercise, researchers have developed a new method of exercise called high-intensity interval training (HIIT) using a combination of speed training and interval training (7). HIIT creates metabolic, structural, ionic, and neural adaptations in muscles. In addition, it increases capillary density, production of vascular growth factors, endothelial proliferation, and FGF and leads to angiogenesis through the process of hypoxia (4). One study evaluated the effect of eight weeks of resistance training on serum myostatin and FGF-2 and demonstrated that training increases serum FGF-2 levels (8). Another study examined the effect of aerobic training on inflammatory factors in the lungs. It was concluded that endurance training is associated with the modulation of inflammatory factors in the lungs, including tumor necrosis factor alpha (TNF- $\alpha$ ) and NF$\kappa \mathrm{B}$, indicating the anti-inflammatory effects of exercise training (9).

In addition to the effective role of exercise training, supplementation plays a strengthening role. Quercetin supplementation has a protective role in the cardiovascular system and belongs to a group of plant pigments called flavonoids. Further, it has the most antioxidant properties among other flavonoids and is even about six times stronger than vitamin C.

Quercetin also neutralizes free radicals and reduces cell damage and death, along with reducing the oxidation of low-density lipoprotein (LDL) by eliminating free radicals, thereby lowering LDL cholesterol and cardiac diseases (10). Studies have shown that this supplement has a positive and significant relationship with the process of angiogenesis and also seems to improve and strengthen the cardiovascular system. On the other hand, it has been found that interval training has a beneficial effect on physiological muscle processes and a positive adaptation to the signaling pathway of the cardiac angiogenesis process.

To the best of our knowledge, no study has so far investigated the effect of interval training on the gene expression of angiogenic factors (NF- $\kappa \mathrm{B}$ and FGF-2) and the relationship between them. Accordingly, this research aimed to explore whether the concurrent use of interval training and quercetin supplementation affects NF- $\kappa B$ and FGF-2 gene expression in patients with MI.

\section{Materials and Methods \\ Animals}

In this applied experimental study, thirty 6-8 week-old male rats, weighing $250 \pm 20 \mathrm{~g}$, were purchased from the Pasteur Institute of Iran and then transferred to the histogenetic laboratory affiliated to the Pasargad Tissue and Gene Company. The animals were kept at $23 \pm 3^{\circ} \mathrm{C}$ with a humidity of $50 \pm 10 \%$ and had ad libitum access to food and water up to the end of the protocol. Their dark-light cycle included 12 hours of dark and 12 hours of light. To create cardiac ischemia, isoprenaline hydrochloride was subcutaneously injected to 30 rats at a dose of $80 \mathrm{mg} / \mathrm{kg}$ ( $0.9 \%$ sodium chloride), and then they were randomly divided into five groups including normal control, MI, MI + training, MI + supplement, and MI + training + supplement each containing six rats. To ensure the induction of experimental MI, a number of rats in MI groups were randomly anesthetized two days after MI, and their cardiac tissue samples were examined using hematochemical eosin staining techniques. Finally, the legitimate animals were included in the study.

\section{Tissue preparation}

The tissue preparation stage included dehydration, clarification, and impregnation. In the dehydration step, the sample was placed in alcohol $70 \%, 80 \%, 90 \%$, and $100 \%$ for 50 minutes each, respectively. The tissue water was absorbed and alcohol was replaced with this action. The sample was then placed in a solution called xylol, which also replaced alcohol.

There were two types of xylol in the clarification phase, each lasting 40 minutes. In the impregnation step, the sample was placed inside the melted paraffin to penetrate into the tissue. Paraffin was solid at room temperature and melted at $70^{\circ} \mathrm{C}$. Up to this stage, it was possible to prepare the tissue both manually in the oven and automatically by a device called autotechnicon. Two types of paraffin were used for 50 minutes each.

After preparation, the tissue was molded by cutting and hydration. At the cutting stage, the samples were cut with a device called microtome at a thickness of 5-10 $\mu \mathrm{m}$. To remove the paraffin inside the sample, the samples were placed inside two types of xylol each for 10 minutes. To stain, the tissue water was slowly returned to xylol according to some steps including keeping alcohol 100\%, $90 \%, 80 \%$, and $70 \%$ and running water for 1 minute each for complete dehydration (11).

\section{Investigation of gene expression}

The concentration and purity of the extracted RNA were evaluated by a nanodrop spectrophotometer. The light absorption of the samples was measured at 260 and 280 
$\mathrm{nm}$, and its concentration was obtained based on the accuracy factor in $\mathrm{ng} / \mu \mathrm{L}$. Next, cDNA synthesis was performed using a cDNA synthesis kit, RNA treated with Dnase I, $1 / 2 \mu \mathrm{L}$ microtube, thermocycler, water treated with DEP, a sampler, and a head sampler.

After measuring the optical density of the samples, the concentrations were reached up to $1 \mathrm{ng} / \mu \mathrm{L}$, to be prepared for cDNA synthesis. Then, $10 \mu \mathrm{L}$ of the treated RNA $\left(D_{\text {nase }}\right.$ at a concentration of $1 \mathrm{ng} / \mu \mathrm{L}$ ) was poured into a 0.2 microtube and $10 \mu \mathrm{L}$ of $\mathrm{cDNA}$ synthesis kit solution was added as well. Next, it was placed in a thermocycler for 5 minutes at $25^{\circ} \mathrm{C}$ followed by 60 minutes at $60{ }^{\circ} \mathrm{C}$. The microtubes were cooled on ice and stored at $-21^{\circ} \mathrm{C}$ for the quantitative polymerase chain reaction ( $\mathrm{qPCR})$. The primer was designed using real-time PCR. The sequence of the forward-reverse primers of genes is presented in Table 1.

Quercetin supplementation with liposome nanoparticles Quercetin supplementation, which was converted to liposome nanoparticles, was daily administered by gavage at a dose of $0.25 \mathrm{mg} / \mathrm{kg}$ body weight for 8 weeks. The first day of quercetin gavage was initially performed 2 days after adaptation to the training environment. During gavage treatment, quercetin nanoliposomal supplementation was performed in two supplement groups at a dose of $10 \mathrm{mg} /$ $\mathrm{kg}$ five days a week at 12 o'clock (12).

\section{Training protocol}

Rats of the training group performed the training protocol for 8 weeks and 5 sessions per week and the other groups were kept in the laboratory condition during the implementation of the protocol. To implement the training protocol, rats in the training and training + supplementation groups were introduced to work on the animal treadmill for two weeks. Then, to determine the intensity of exercise training, the maximum speed test was performed on the treadmill so that after 10-20 minutes of warm-up at $40-50 \% \mathrm{VO}_{2 \max }$ intensity, the treadmill speed increased $0.03 \mathrm{~m} / \mathrm{s}$ every two minutes until the animal was unable to run anymore. The speed was considered as the speed of $\mathrm{VO}_{2 \max }$ at which blood lactate levels were above $6 \mathrm{mmol} / \mathrm{L}$

According to Table 2, the main training protocol as HIIT was performed on an animal treadmill (Tajhiz Gostar, 2016, Tehran, Iran) in the training group. The HIIT protocol consisted of a 10-minute warm-up period (50-55\% maximal oxygen uptake $\mathrm{VO}_{2 \max }$ ), 7 interval training periods (4-minute intervals at $80 \%-90 \% \mathrm{VO}_{2 \max }$ interspersed with 3 -minute periods of $\left.65-75 \% \mathrm{VO}_{2 \max }\right)^{2 \max }$, and a 5-minute cooldown period. It should be noted that no training shock was used during the training program and if necessary, the animals were forced to continue training using their hands or creating a sound stimulus on the cap of the treadmills (13).

\section{Statistical data analysis}

SPSS statistical (version 23) and GraphPad Prism (version 6.0) software were used to analyze the data and to draw the graphs, respectively. Moreover, the Kolmogorov-Smirnov test was applied to determine the normality of the data. Eventually, one-way analysis of variance (ANOVA) and Tukey's post hoc test at a significance level of $<0.05$ were employed to analyze the data.

Results

Considering the findings of the present study, the intergroup comparison of the expression of the two studied genes are provided in Table 3.

\section{$N F-\kappa B$ gene expression}

The results of one-way ANOVA showed a significant difference between different groups of the present study $(\mathrm{F}=16.02, P=0.001)$.

The results of Tukey's post hoc test revealed that NF- $\kappa \mathrm{B}$ levels in the MI group significantly increased compared to the control group $(P=0.001)$. However, NF- $\kappa \mathrm{B}$ levels significantly decreased in the $\mathrm{MI}+$ training $(P=0.001)$, $\mathrm{MI}+$ supplement $(P=0$. 001), and $\mathrm{MI}+$ training + supplement $(P=0.001)$ groups in comparison with the MI group. The statistical results of this gene expression

Table 1. Sequence of primers

\begin{tabular}{ll}
\hline Gene Name & Oligo Sequence \\
\hline NF- $\kappa B$ & F AGAAGGCTGGAGAAGATGAG \\
& R TTGGTGCCTCGTGTCTTCTGT \\
FGF-2 & F AGTTGTGTCCATCAAGGGAGTGTG \\
& R ATACTGCCCAGTTCGTTTCAGTGC \\
GAPDH & R AGGTCGGTGTGAACGGATTTG \\
\hline
\end{tabular}

Note. NF-кB: Nuclear factor kappa B; FGF: Fibroblast growth factor; GAPDH: Glyceraldehyde-3-phosphate dehydrogenase.

Table 2. Eight weeks of HIIT training for MI

\begin{tabular}{lcccc}
\hline Weeks & Type of Activity & $\begin{array}{c}\text { Running Speed } \\
(\mathbf{m} / \mathbf{m i n})\end{array}$ & $\mathbf{V o}_{\mathbf{2}_{\text {max }}}(\%)$ & $\begin{array}{c}\text { Duration } \\
(\mathbf{m i n})\end{array}$ \\
\hline \multirow{2}{*}{ 1-8 } & 4-minute intervals & 25 & $80-90$ & 49 \\
& 3-minute intervals & 15 & $65-75$ & \\
\hline
\end{tabular}

Note. HIIT: High-intensity interval training; MI: Myocardial infarction.

Table 3. One-way ANOVA for NF-kB mRNA and FGF-2 mRNA

\begin{tabular}{lcccccc}
\hline Group Variable & Control & MI & MI + Training & MI + Supplement & MI + Training + Supplement & $\boldsymbol{P}$ value \\
\hline NF-кB & $0.055 \pm 0.006$ & $0.126 \pm 0.026$ & $0.036 \pm 0.018$ & $0.053 \pm 0.011$ & $0.046 \pm 0.001$ \\
FGF-2 & $0.009 \pm 0.005$ & $0.002 \pm 0.0008$ & $0.003 \pm 0.0007$ & $0.004 \pm 0.001$ & $0.006 \pm 0.002$ \\
\hline
\end{tabular}

Note. ANOVA: Analysis of variance; NF-кB: Nuclear factor kappa B; FGF-2: Fibroblast growth factor 2; MI: Myocardial infarction. 
are illustrated in Figure 1.

\section{FGF-2 gene expression}

The results of one-way ANOVA showed that there was a significant difference between the different groups of the present study $(P=0.04, \mathrm{~F}=3.73)$. The results of Tukey's post hoc test showed that the levels of FGF2 gene expression were significantly lower only in the control group compared to the MI group $(P=0.007)$. The statistical results of this gene are shown in Figure 2.

\section{Discussion}

This study mainly aimed to evaluate the effect of eight weeks of HIIT and quercetin nanoliposome supplementation on NF- $\mathrm{BB}$ and FGF-2 gene expression in the heart tissue of MI rats.

The results showed that the levels of NF- $\kappa \mathrm{B}$ gene expression in the control group were significant and relative to the MI group, the MI group relative to the training + MI group, the MI group relative to the supplementation + MI group, and the MI group relative to the supplementation + training + MI group. On the other hand, the levels of FGF-2 gene expression were significant only in the control group compared to the MI group.

MI is the most common cause of heart failure, which leads to left ventricular dysfunction. Therefore, it seems necessary to study the physiological mechanisms associated with this disease and find a suitable solution mainly to prevent this complication and then to treat this disease.

Exercise is suggested as part of the rehabilitation program for patients with MI. It alone has many positive effects on the cardiovascular system, including improving heart function and parallel circulation of the coronary artery. In addition, exercise and muscle contractions provide a powerful stimulus for the regeneration of the vascular structure.

The velocity of the blood flow in the vessel increases following an increase in the shear stress of the vessel. This increase in shear stress is the main stimulus for the enlargement of ductal and capillary vessels by angiogenesis. Stimulation-induced angiogenesis due to exercise includes angiogenic factors such as VEGF and FGF-2. It has been suggested that FGF-2 plays an important role in exercise-induced cardiac angiogenesis (14). Consistent with the results of the present study, a study was conducted on the elderly to explore the effect of 8 weeks of running on angiogenic changes and molecular mediators associated with myocardial angiogenesis. The results demonstrated that the $\mathrm{NF}-\kappa \mathrm{B}$ myocardium of the elderly group significantly increased compared to the younger group, and the exercise group indicated a significant increase in NF- $\kappa \mathrm{B}$ gene expression (15).

Another study compared the effects of two types of HIIT and moderate-intensity continuous training (MICT) on NF- $\kappa B$ and IL-6 gene expression in the lung tissue of young and old rats. Based on the results, there was a

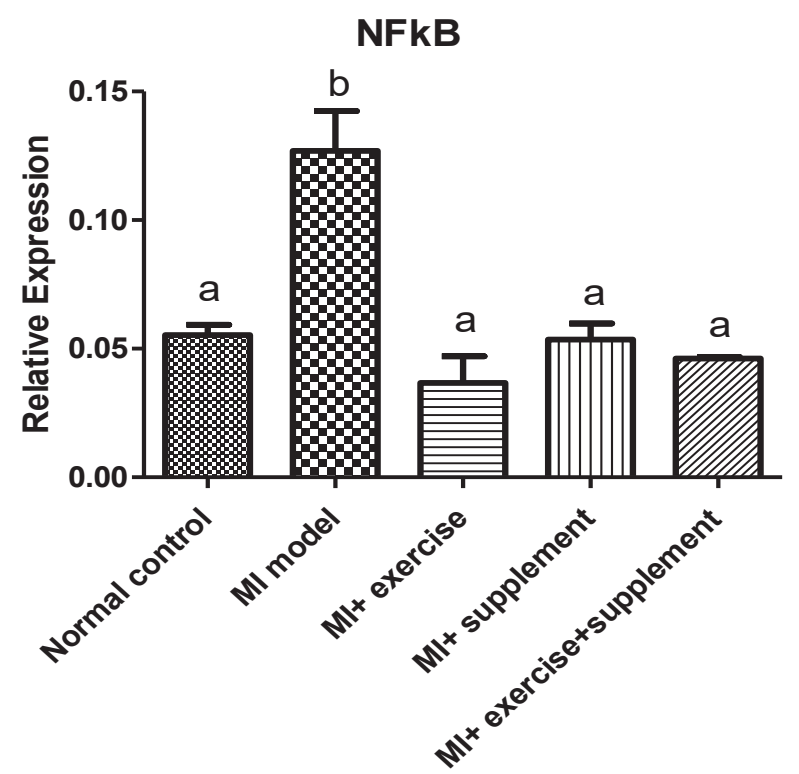

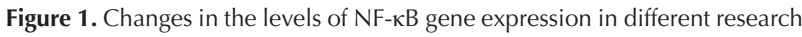
groups. Note. NF-kB: Nuclear factor kappa B; MI: Myocardial infarction. Dissimilar letters at the top of each bar indicate significant changes between different research groups $(P=.0001)$. Similar letters at the top of each bar represent a significant change $(P=0.001)$.

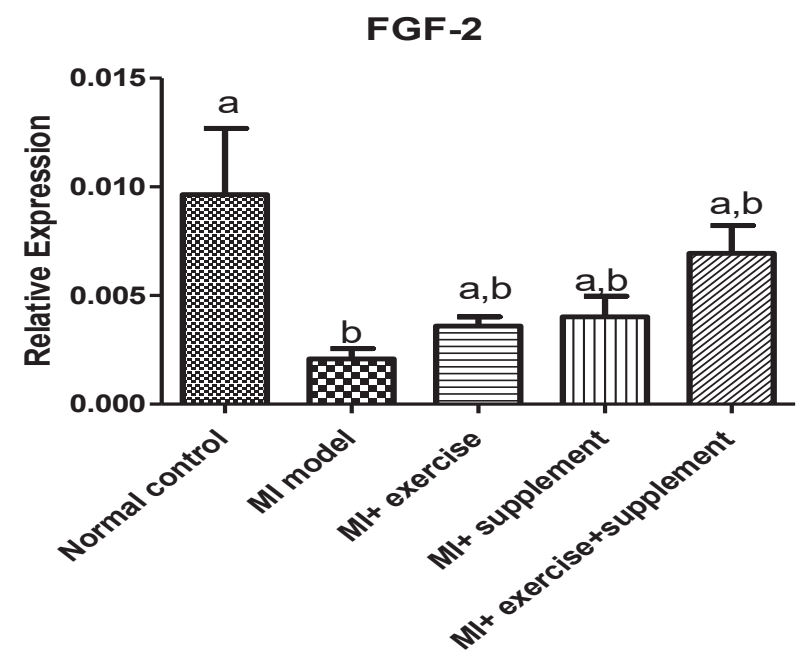

Figure 2. Changes in the levels of FGF-2 gene expression in different research groups. Note. FGF-2: Fibroblast growth factor 2; MI: Myocardial infarction. Dissimilar letters at the top of each bar denote significant changes between different research groups $(P=0.04)$. Similar letters at the top of each bar demonstrate a significant change $(P=0.007)$.

significant difference in NF- $\kappa \mathrm{B}$ gene expression between the old control and old continuous training groups, between the old control and old HIIT, as well as between the old continuous training and old HIIT groups (16).

Regarding FGF-2 gene expression, there are analogous studies with the present study. For example, a study investigated the effect of ten HIIT on the levels of some angiogenesis factors and pulmonary function in men with prostate cancer. The results showed that HIIT significantly increased FGF levels in the experimental group compared to the control group (4).

Some conflicting studies evaluated the effects of HIIT and MICT on the levels of FGF-2 gene expression in the visceral and subcutaneous adipose tissue of male 
Wistar rats. The results represented that both types of training interventions significantly increased FGF-2 gene expression in the subcutaneous adipose tissue (5).

A similar study examined the effect of endurance training on the process of angiogenesis in the soleus muscle of rats with MI but reported no changes in FGF-2 gene expression (17).

It should be noted that endurance training was used with no supplement in the last two studies. Further, the FGF-2 gene was studied in a target tissue other than the myocardium. The possible mechanism of gene expression may be different in other tissues.

To investigate the effect of supplementation, performing 8 weeks of HIIT and quercetin supplementation on the dimensional and functional adaptations of the left ventricle in men with hypertension caused a significant increase in left ventricular systolic and diastolic dimensions in the HIIT and quercetin supplementation group (18).

Quercetin induces endothelial nitric oxide synthase phosphorylation, thereby increasing nitric oxide release, improving cardiac flow, and paving the way for angiogenetic processes. Quercetin also plays an important role in reducing the formation of inflammatory molecules and inhibiting their activity by inhibiting NF- $\mathrm{B}$ transcription and other proinflammatory signaling cascades (19). There are two main pathways for activating NF- $\kappa \mathrm{B}$, including classical and non-classical pathways. Activation via the classical pathway occurs when an external ligand binds to a receptor on the surface of the membrane, resulting in oligomerization, using a number of receptive molecules, and finally, activating the ubiquitin-dependent TAK1-IKK complex. IKK mediates IKBa phosphorylation at Ser32 and Ser36 levels, where I $\kappa \mathrm{B} \alpha$ is targeted for proteasomal degradation, and ultimately, more rapidly transmits NF$\mathrm{\kappa B}$ to the nucleus. In the non-classical pathway, ligand binding leads to the activation of NF- $\kappa \mathrm{B}$-stimulated kinase and IKKa, causing the processing of the p100 pathway and the transfer of $\mathrm{p} 52 /$ RelB into the nucleus.

MI occurs under conditions of the lack of blood and oxygen delivery to the heart cells, thus it has been reported that hypoxia-induced NF- $\kappa \mathrm{B}$ is caused by the phosphorylation of the tyrosine residue $I \kappa B a$, and this activation does not lead to the breakdown of I $\mathrm{BB}$. Although it indicates an IKK-independent mechanism, NF- $\kappa \mathrm{B}$ is one of the important adaptation factors in response to hypoxia. The underlying mechanism of this activation has not been defined yet (20).

The use of a specific inhibitor of PI 3-kinase or IRAK revealed that IRAK activates PI 3-kinase, its signaling phosphorylates $I K K \alpha / \beta$, and degrades I $\mathrm{B}$, thereby leading to NF- $\kappa B$ activation. The stimulation of FGF- 2 by IL- $1 \beta$ is completely blocked by NF- $\mathrm{BB}$ (sulfasalazine) or PI 3-kinase inhibitors. Chromatin safety deposition also shows that $N F-\kappa B$ is an FGF-2 transcription factor because NF- $\kappa B$ binds to the NF- $\kappa \mathrm{B}$ domain site of the FGF-2 promoter. Based on these data, IL- $1 \beta$ signaling is a combination of the classical pathway and PI 3-kinase signaling, which is essential for resetting FGF-2 production via NF- $\mathrm{kB}$ and plays a key role as an FGF-2 transcription factor (21).

The therapeutic effect of FGF-2 in response to inflammation caused by acute lung disease in rats was investigated to achieve possible mechanisms regarding health. The results showed that FGF-2 treatment reduced inflammatory responses, pulmonary capillary leakage, and lung damage, thereby improving survival and persistence in rats with pulmonary inflammations. Therefore, endothelial lesions and macrophage inflammations stimulated by lipopolysaccharide are inhibited by FGF-2 through the AKT/P38/NF- $\kappa$ B signaling pathway (22).

A study evaluated the role of the JAK/STAT signaling pathway in the incidence of acute MI in rats and then focused on the effect of this pathway on the expression of the nuclear factor (NF- $\kappa \mathrm{B})$ in the myocardium of rats with MI. It was found that the JAK/STAT signaling pathway was involved in the onset of MI and may also be involved in left ventricular regeneration after MI. The involvement of the JAK/STAT signaling pathway at the onset of MI may be associated with its effects on NF- $\kappa B$ and TNF- $\alpha$ expression (23).

It has also been suggested that BFGF or FGF-2, through the JAK/STSTE/RAS protein pathway, stimulate processes such as cell proliferation, differentiation, adhesion, and migration during angiogenesis (24). According to the results of the present study, interval training with quercetin consumption at the beginning of MI leads to increased levels of FGF-2 and NF- $\kappa$ B gene expression and the process of angiogenesis. In the advanced stages of MI, these two variables reduce the levels of NF- $\kappa B$ gene expression by inhibiting the ubiquitin-dependent TAK1IKK complex, which inhibits FGF-2 gene transcription. As a result, they control inflammatory reactions and angiogenesis processes by reducing inflammatory responses. In this study, there was a short time to start training after the induction of infarction. Furthermore, the concurrent implementation of training and gavage in one day, as well as the lack of time to rest were stress factors for sick rats and can be considered as some of the limitations of the study. It is suggested that further studies be performed to achieve the NF- $\mathrm{kB}$ gene activating signaling pathway with quercetin supplementation and interval training. For this purpose, the induction method of infarction could be changed and the surgical method could be used instead. Likewise, longer training protocols should be implemented if possible.

\section{Conclusion}

Based on the obtained results, FGF-2 could inhibit or reduce $\mathrm{MI}$ inflammations in progressive conditions. On the other hand, the role of supplementation and training was highlighted in reducing NF- $\kappa \mathrm{B}$ gene expression in $\mathrm{MI}$, and it was found that NF- $\mathrm{BB}$ gene expression is activated through FGF-2 in the MI group.

As mentioned earlier, there are two pathways for NF- $\kappa B$ gene activation, including the AKT/P38/NF- $\kappa \mathrm{B}$ and JAK/ 
STAT signaling pathways. Therefore, HIIT and quercetin supplementation in the progressive condition can, alone or concurrently, reduce NF- $\kappa \mathrm{B}$ gene expression from the JAK/STAT signaling pathway, which is involved in the onset of MI.

\section{Acknowledgments}

This study was derived from a thesis in sports physiology (no: 1754846201554971399162328958.) approved by the Physical Education and Sports Sciences Department of Isfahan (Khorasgan) Branch, Islamic Azad University, Isfahan, Iran in July 2021. The authors of this article express their gratitude to all the dear friends and colleagues who helped us in this research.

\section{Authors' Contribution}

SZ performed tests and collected the data. $\mathrm{KH}, \mathrm{FT}$, and $\mathrm{AH}$ designed the study and analyzed the data. SZ and $\mathrm{KH}$ wrote and revised the manuscript. All authors approved the final version of the manuscript.

\section{Conflict of Interests}

The authors of the article have no conflict of interests to disclose.

\section{Ethical Approval}

The study protocol was approved by the Research Ethics Committee of Isfahan (Khorasgan) Branch, Islamic Azad University, Isfahan, Iran (IR.IAU.KHUISF.REC.1400.092).

\section{Funding/Support}

This study received no funds.

\section{References}

1. Rajadurai M, Stanely Mainzen Prince P. Preventive effect of naringin on cardiac markers, electrocardiographic patterns and lysosomal hydrolases in normal and isoproterenolinduced myocardial infarction in Wistar rats. Toxicology. 2007;230(2-3):178-88. doi: 10.1016/j.tox.2006.11.053.

2. Akbari H, Choobineh S, Akbarnejad A, Naderi N. The effect of eight weeks of high intensity interval training on genes expression of eNOS, VEGF and protein expression of CD31 in myocardial infarction. Journal of Knowledge \& Health. 2019;14(3):16-22. doi: 10.22100/jkh.v14i3.2287. [Persian].

3. Nourshahi M, Taheri C, Rajbar K. Stimuli of angiogenesis during exercise and physical activity. Ofogh-e-Danesh. 2012;18(5). [Persian].

4. Fathollahi Shoorabeh F, Faramarzi M, Hemmati R. The Effect of 10 weeks of high-intensity exercise training on resting levels of some angiogenesis and pulmonary function of men with prostate cancer. J Fasa Univ Med Sci. 2019;8(4):1097-105. [Persian].

5. Mirzaei Amirabadi G, Asad MR, Rahimi M. The effect of exercise with different intensity and volume on fibroblast growth factor (FGF-2) gene expression in subcutaneous and visceral adipose tissue in male rats. J Sport Exerc Psychol. 2018;11(2):97-108. [Persian].

6. Sharifat N, Jafari-Hafshejani F, Dayati P, Lorestanpoor P, Paydar A, Babaahmadi Rezaei H. Inhibitory effect of curcumin on phosphorylation NFkB-p65 induced by hydrogen peroxide in bovine endothelial cells. J Fasa Univ Med Sci. 2017;7(2):28390. [Persian].

7. Bayati M, Gharakhanlou R, Ghobadi H, Nikkhah M, Farzad B. Different responses of serum VEGF in trained and untrained men to high-intensity interval exercise. J Appl Exerc Physiol. 2016;12(23):64-75. [Persian].

8. Khadivi Borujeni Z, Rajabi H, Marandi SM, Haghjoo SH, Khadivi Borujeni A, Nourian E. Effect of resistance training on plasma FGF-2 and myostatin level in male Wistar rats. Res Sports Med Technol. 2018;8(15):11-22. [Persian].

9. Fashi M, Agha-Alinejad H, Asilian Mahabadi H, Rezaei B,
Pakrad B, Rezaei S. The effects of aerobic exercise on NF-kB and TNF- $\alpha$ in lung tissue of male rat. Novelty in Biomedicine. 2015;3(3):131-4. doi: 10.22037/nbm.v3i3.8001.

10. Mahmoudi K, Moonikh K, Yazdani F. Effect of quercetin supplementation on CRP and IL-6 after acute exhaustive resistance training in young men. J Anim Physiol Dev. 2019;12(44):47-57. [Persian].

11. Omomi M, Taghian F, Sharifi GR. The effect of six weeks of aerobic exercise training alone and along with consumption of ginger extract encapsulated in chitosan nanoparticles on changes in heart tissue morphology and expression level of mitogen-activated protein kinase (MAPK) gene in rats with myocardial infarction: an experimental study. Stud Med Sci. 2021;32(1):1-13. [Persian].

12. Cui W, Hu G, Peng J, Mu L, Liu J, Qiao L. Quercetin exerted protective effects in a rat model of sepsis via inhibition of reactive oxygen species (ROS) and downregulation of high mobility group box 1 (HMGB1) protein expression. Med Sci Monit. 2019;25:5795-800. doi: 10.12659/msm.916044.

13. Wang B, Zhou R, Wang Y, Liu X, Shou X, Yang Y, et al. Effect of high-intensity interval training on cardiac structure and function in rats with acute myocardial infarct. Biomed Pharmacother. 2020;131:110690. doi: 10.1016/j.biopha.2020.110690.

14. Shen M, Yu M, Qiu C, Zhang G, Li J, Fang W, et al. Myocardial angiogenesis induced by exercise training involves a regulatory mechanism mediated by kinin receptors. Clin Exp Hypertens. 2021;43(5):408-15. doi: 10.1080/10641963.2021.1896725.

15. Pourheydar B, Biabanghard A, Azari R, Khalaji N, Chodari L. Exercise improves aging-related decreased angiogenesis through modulating VEGF-A, TSP-1 and p-NF-Kb protein levels in myocardiocytes. J Cardiovasc Thorac Res. 2020;12(2):12935. doi: 10.34172/jcvtr.2020.21.

16. Motiei Haghighi M, Soori R, Shabkhiz F, Choobineh S. Comparison of the effect of high intensity interval training and moderate intensity continuous training on gene expression of $\mathrm{I}-6$ and $\mathrm{Nf}-\mathrm{Kb}$ in lung tissue of old and young male rats. J Ilam Univ Med Sci. 2019;27(5):54-64. doi: 10.29252/ sjimu.27.5.54. [Persian].

17. Ardakanizade M, Ranjbar K, Nazem F. The effect of ten factors of endurance activity on the gene expression of factors involved in the process of skeletal muscle angiogenesis after myocardial infarction in rats. Koomesh. 2017; 19(1):84-92.

18. Kashef M, Mahmoudi K, Salehpour M, Moonikh K. The effect of high-intensity interval training (HIIT) and quercetin supplementation on dimension and functional left ventricular adaptations in men with hypertension and CAD after PCl. Daneshvar Medicine. 2020;27(144):35-48. [Persian].

19. Chekalina NI, Shut SV, Trybrat TA, Burmak YH, Petrov YY, Manusha YI, et al. Effect of quercetin on parameters of central hemodynamics and myocardial ischemia in patients with stable coronary heart disease. Wiad Lek. 2017;70(4):707-11.

20. Culver C, Sundqvist A, Mudie S, Melvin A, Xirodimas D, Rocha S. Mechanism of hypoxia-induced NF-kappaB. Mol Cell Biol. 2010;30(20):4901-21. doi: 10.1128/mcb.00409-10.

21. Lee JG, Kay EP. NF-KB is the transcription factor for FGF-2 that causes endothelial mesenchymal transformation in cornea. Invest Ophthalmol Vis Sci. 2012;53(3):1530-8. doi: 10.1167/ iovs.11-9102.

22. Pan $\mathrm{X}, \mathrm{Xu} \mathrm{S}, \mathrm{Zhou} \mathrm{Z}$, Wang F, Mao L, Li H, et al. Fibroblast growth factor-2 alleviates the capillary leakage and inflammation in sepsis. Mol Med. 2020;26(1):108. doi: 10.1186/s10020-020-00221-y.

23. Zhang S, Liu X, Goldstein S, Li Y, Ge J, He B, et al. Role of the JAK/STAT signaling pathway in the pathogenesis of acute myocardial infarction in rats and its effect on NF- $\mathrm{kB}$ expression. Mol Med Rep. 2013;7(1):93-8. doi: 10.3892/mmr.2012.1159.

24. Karnoub AE, Weinberg RA. Ras oncogenes: split personalities. Nat Rev Mol Cell Biol. 2008;9(7):517-31. doi: 10.1038/ nrm2438. 